\title{
ENDOCRINOLOGY
}

\section{Localization of an Insulinoma by Endoscopic Ultrasound: A Case Report and Review of Diagnostic Modalities}

\author{
Kristen Jackson, MD, Joseph Yoo, MD, and Bolin Niu, MD
}

\begin{abstract}
Insulinomas are a type of pancreatic neuroendocrine tumor ( $\mathrm{PNET}$ ) that arises from pancreatic endocrine tissue. They are rare, with an estimated incidence of 4 cases per 1 million person-years? ${ }^{7}$. While typically larger pNETs are detectable using conventional imaging studies such as transabdominal ultrasound, CT, or MRI, insulinomas are frequently missed by conventional imaging due to their relatively small size. We present the case of a 66-year-old African American male with an insulinoma undetected by contrast-enhanced MRI, that was ultimately localized and diagnosed via endoscopic ultrasound (EUS) with fine needle aspiration (FNA).
\end{abstract}

\section{CASE PRESENTATION}

A 66-year-old African American male with past medical history of hypertension, obstructive sleep apnea, glaucoma, coronary artery disease status post coronary stents, and squamous cell carcinoma of the larynx status post total laryngectomy presented from a rehab facility with weakness, lethargy, and repeated episodes of hypoglycemia. The patient denied history of diabetes, use of oral antihyperglycemic agents, or insulin.

On arrival, the patient's glucose level was $35-45 \mathrm{mg} / \mathrm{dL}$ on repeated testing; he was started on $10 \%$ dextrose (D10) intravenous drip to maintain normoglycemia. Fasting laboratory evaluation (Table 1) was remarkable for elevated levels of insulin, c-peptide, and proinsulin. TSH and ACTH levels were within normal limits. Morning cortisol level at 8am was low at 10.9 mcg/dL; however, cosyntropin stimulation test showed adequate response of cortisol. The patient also had elevated C-reactive protein, low ionized calcium, and a negative insulin autoantibody assay (Table 1).

MRI of the abdomen with and without gadolinium contrast and magnetic resonance cholangiopancreatography (MRCP) were performed, demonstrating normal enhancement of the pancreas without masses, a normal pancreatic duct, and no biliary ductal dilatation. Somatostatin-receptor scintigraphy was negative. EUS was performed given continued high clinical suspicion of an insulin-producing tumor, and revealed a $1.2 \times 0.8$ $\mathrm{cm}$ round hypoechoic lesion in the pancreatic head (Figure 1). FNA cytology showed immunohistochemical stains positive for neuroendocrine tumor markers chromogranin and synaptophysin, and a Ki67 proliferation index less than $1 \%$; the stains were negative for insulin. The patient was started on subcutaneous octreotide with ongoing D10 drip.

The patient was taken to the operating room; however, resection of the tumor via pancreaticoduodenectomy was aborted secondary to the patient's instability and pressor requirement intraoperatively. Instead, alcohol ablation of the lesion was performed under ultrasound guidance. By post-procedure day 4, the patient was able to maintain normoglycemia off D10 drip and octreotide.

\section{DISCUSSION}

Insulinomas are a type of pNET that arises from pancreatic endocrine tissue. There are ten commonly recognized pNETs, the most common being insulinomas, with an estimated incidence of 4 cases per 1 million person-years ${ }^{7.14}$. Presenting symptoms in about $90 \%$ of patients include neuroglycopenia, manifesting as confusion, altered consciousness, forgetfulness, or coma. Less often, symptoms of sympathetic over-stimulation such as sweating, tremors, and palpitations occur in 60-70\% of patients 9 . Symptoms typically occur during fasting, delayed meals, or exercise ${ }^{5}$. Timing of symptoms is important in distinguishing insulinomas from other forms of hyperinsulinism such as nesidioblastosis, an acquired form of hyperinsulinism due to beta cell hyperplasia that typically presents with postprandial hypoglycemia following bariatric surgery 9 . According to the Endocrine Society clinical practice guidelines, a patient with fasting or postprandial endogenous hyperinsulinemic hypoglycemia, defined as signs or symptoms of hypoglycemia with plasma concentrations of glucose $<55 \mathrm{mg} / \mathrm{dl}$, plasma insulin at least $3.0 \mathrm{\mu U} / \mathrm{ml}$, C-peptide of at least $0.6 \mathrm{ng} / \mathrm{ml}$, proinsulin of at least $5 \mu \mathrm{M}$, a negative screening for oral hypoglycemic agents, and no circulating insulin antibodies, should undergo procedures to localize an insulinoma ${ }^{5}$. The guidelines specify that localization should be performed using CT, MRI, transabdominal or endoscopic ultrasound, or, if necessary, selective pancreatic arterial calcium injections with measurements of hepatic venous insulin levels.

Treatment of insulinomas is directed at the hyperinsulinemic state and the insulinoma itself. Surgical 
resection is the treatment of choice and is successful in greater than $95 \%$ of cases. Fewer than $10 \%$ of insulinomas are malignant at the time of diagnosis. Rates of recurrence are $7 \%$ for those without the associated MEN-1 syndrome and $21 \%$ for those with MEN-15,9. Medical treatment is used preoperatively and for those with unresectable insulinomas. First line medical therapy is small, frequent feedings, and diazoxide: a benzothiadiazine that directly inhibits insulin release ${ }^{9}$. In recent years, endoscopic ultrasound-guided ethanol ablation has emerged as a less invasive treatment option with fewer post-procedural complications, especially in elderly patients or other poor surgical candidates ${ }^{16}$.

Because surgical resection is the treatment of choice, preoperative localization and staging are extremely important. Imaging techniques such as transabdominal ultrasound, CT, or MRI, are typically used to localize insulinomas. Studies have shown that detection of pNETs using conventional imaging is dependent on the lesion size, with detection rates less than $10 \%$ for tumors smaller than $1 \mathrm{~cm}$ in diameter, $30-40 \%$ for tumors $1-3 \mathrm{~cm}$ in diameter, and greater than 50\% for those larger than $3 \mathrm{~cm}$ in diameter ${ }^{4}$. Insulinomas have been inconsistently localized by conventional imaging compared to other pNETs because they are relatively smaller at the time of diagnosis, with $90 \%$ measuring less than $2 \mathrm{~cm}$ in diameter ${ }^{11}$. Despite advances in technology and improved detection rates compared to older studies, the sensitivities of CT and MRI are still below $80 \%^{12,17}$. Finally, transabdominal ultrasound has been reported to have a detection rate of only $9-22 \%, 17$.

Conversely, EUS has been shown to be able to detect pancreatic lesions as small as $0.3-0.5 \mathrm{~cm}$ in diameter ${ }^{2.4}$. Furthermore, EUS has been shown to successfully localize 92\% of CT-negative pNETs, which includes insulinomas and non-insulinomas ${ }^{12}$. However, some studies have demonstrated lower detection rates and propose that sensitivity is likely variable depending on the experience of the endoscopist ${ }^{11}$. A retrospective review of 237 cases reported an EUS sensitivity of only $75 \%$ and described superior sensitivity (93\%) with selective arterial calcium stimulation testing (SACST) ${ }^{15}$. SACST is another invasive diagnostic modality that utilizes hepatic venous sampling of insulin after the injection of calcium, which stimulates beta cells to release insulin, c-peptide, and proinsulin.

In addition to often being more reliable in the detection and localization of insulinomas compared to conventional imaging, EUS also uniquely allows for diagnostic material to be obtained in a safe and minimally invasive manner via FNA, can aid surgical resection by preoperatively tattooing an otherwise undetected insulinoma, and has shown therapeutic potential via ultrasound guided ethanol ablation 4, 10,13. Further, EUS as a method of preoperative localization of pancreatic neuroendocrine tumors is cost effective. In a case-control study matching patients with preoperative EUS versus those who underwent surgical exploration without EUS, the EUS group had reduced charges for preoperative localization studies: $\$ 2620$ versus $\$ 4846$ per patient $(p<0.05)^{3}$. The difference was attributed to reductions in the number of venous sampling procedures and diagnostic angiograms performed. The study also noted decreased surgical and total anesthesia times in patients that underwent EUS localization prior to surgery.

EUS, with or without FNA, is a safe, minimally invasive, and reliable method by which to localize insulinomas and other pNETs, and offers additional value in diagnostic and therapeutic options. As some physicians have already proposed, EUS should be considered as a first choice among all techniques used to investigate insulinomas and other pNETs p $^{2,6}$.

\section{REFERENCES}

1. Alsohaibani F, Bigam D, Kneteman N, et al. The impact of preoperative endoscopic ultrasound on the surgical management of pancreatic neuroendocrine tumours. Can J Gastroenterol 2008;22:817-20.

2. Anderson MA, Carpenter S, Thompson NW, et al. Endoscopic ultrasound is highly accurate and directs management in patients with neuroendocrine tumors of the pancreas. Am J Gastroenterol. 2000;95:2271-2277.

3. Bansal, Rajiv, William Tierney, Steven Carpenter, Norman Thompson, and James M. Scheiman. "Cost Effectiveness of EUS for Preoperative Localization of Pancreatic Endocrine Tumors." Gastrointestinal Endoscopy 49.1 (1999): 19-25.

4. Chang F, Chandra A, Culora G, Mahadeva U, Meenan J, Herbert A. Cytologic diagnosis of pancreatic endocrine tumors by endoscopic ultrasound-guided fine-needle aspiration: a review. Diagnostic Cytopathology. 2006;34(9):649-658

5. Cryer PE, Axelrod L, Grossman AB, Heller SR, Montori VM, Seaquist ER, et al. Evaluation and management of adult hypoglycemic disorders: an Endocrine Society Clinical Practice Guideline. J Clin Endocrinol Metab. 2009:94:709-728.

6. De Angelis C, Carucci P, Repici A, et al. Endosonography in decision making and management of gastrointestinal endocrine tumors. Eur $\mathrm{J}$ Ultrasound 1999;10:139-150.

7. Grant C. Insulinoma. Best Practice and Research Clinical Gastroenterology. 2005;19(5):783-798.

8. Guettier J, Kam A, Chang R, Skarulis M, Cochran C, Alexander HR, Libutti S, Pingpank J, Gorden P. Localization of insulinomas to regions of the pancreas by intraarterial calcium stimulation: The NIH experience. J Clin Endocrinol Metab. 2009 Apr;94(4): 1074-1080

9. Ito $T$, Igarashi $H$, et al. Pancreatic neuroendocrine tumors: clinical features, diagnosis and medical treatment: advances. Best Pract Res Clin Gastroenterol. 2012 December ; 26(6): 737-753.

10. Jurgensen C, Schuppan D, Neser F, Ernstberger J, Junghans U, Stolzel U. US-guided alcohol ablation of an insulinoma. Gastrointestinal Endoscopy. 2006;63(7): 1059-1062

11. Kann, P. H., M. Rothmund, and A. Zielke. "Endoscopic Ultrasound Imaging of Insulinomas: Limitations and Clinical Relevance." Experimental and Clinical Endocrinology \& Diabetes 113.8 (2005): 471-74.

12. Khashab MA, Yong E, Lennon AM, Shin EJ, Amateau S, Hruban RH, Olino K, Giday S, Fishman EK, Wolfgang CL, Edil BH, Makary M, Canto MI. EUS is still superior to multidetector computerized tomography for detection of pancreatic neuroendocrine tumors. Gastrointest Endosc. 2011 Apr;73(4):691-696.

13. Levy M, Thompson G, Topazian M, Callstrom M, Grant C, Vella A. US-guided ethanol ablation of insulinomas: a new treatment option. Gastrointestinal Endoscopy. 2012; 75(1): 200-206.

14. Metz DC, Jensen RT. Gastrointestinal neuroendocrine tumors: pancreatic endocrine tumors. Gastroenterology. 2008;135(5):1469.

15. Placzkowski, K.A., et al., Secular Trends in the Presentation and Management of Functioning Insulinoma at the Mayo Clinic, 1987-2007. The Journal of Clinical Endocrinology \& Metabolism, 2009. 94(4): p. 1069-1073.

16. Qin, Shan-Yu, Xiu-Ping Lu, and Hai-Xing Jiang. "EUS-Guided Ethanol Ablation of Insulinomas." Medicine (Baltimore) 93.14 (2014)

17. Wei, J., et al., Diagnosis and surgical management of insulinomas in 33 consecutive patients at a single institution. Langenbeck's Archives of Surgery, 2016. 401(7): p. 1019-1025. 